Highly Energetic Physical Processes and

Mechanisms for Emission from Astrophysical Plasmas

IAU Symposium, Vol. 195, 2000

P. C. H. Martens, S. Tsuruta, and M. A. Weber, eds.

\title{
The Constellation X-ray Mission
}

\author{
N. E. White \\ NASA Goddard Space Flight Center, Code 662, Greenbelt, MD 20771, \\ U.S.A.
}

H. Tananbaum

Harvard-Smithsonian Center for Astrophysics, 60 Garden St, Cambridge, MA 02138, U.S.A.

\begin{abstract}
The Constellation-X mission is a large collecting-area X-ray facility emphasizing observations at high spectral resolution $(E / \Delta E \sim$ $300-3000)$ while covering a broad energy band $(0.25-40 \mathrm{keV})$. By increasing the telescope aperture and utilizing efficient spectrometers, the mission will achieve a factor of 100 increased sensitivity over current highresolution $\mathrm{X}$-ray spectroscopy missions. The use of focusing optics across the 10-40 keV band will provide a similar factor of 100 increased sensitivity in this band. When observations commence in $\sim 2008$, Constellation$\mathrm{X}$ will address many pressing questions concerning the extremes of gravity and the evolution of the Universe.
\end{abstract}

\section{Introduction}

The Constellation X-ray mission is a central element in NASA's Structure and Evolution of the Universe (SEU) program to explore the cycles of matter and energy in the evolving Universe and to probe black holes, the ultimate limits of gravity. Constellation- $\mathrm{X}$ combines both the large collecting area and sensitive spectrometers required to obtain high spectral resolution, broad bandpass (0.25-40 keV) spectroscopy for all classes of X-ray sources, over a wide range of luminosity and redshift. It is the X-ray equivalent of the Keck Telescope, and, as such, will represent a major advance in sensitivity, providing up to a factor of 100 increase in throughput over currently planned high-resolution $(R>300)$ $\mathrm{X}$-ray spectroscopy missions. We review here the mission design and a selection of the key science goals that the mission will address.

\section{The Mission}

The $0.25-10 \mathrm{keV} \mathrm{X}$-ray band contains the K-shell lines for all of the abundant metals (carbon through zinc) and the L-shell lines of many. The detailed Xray line spectra are rich in plasma diagnostics which also provide unambiguous constraints on physical conditions in the sources. A spectral resolving power of at least 300 is required to separate the He-like density-sensitive triplet. In the region near the iron $\mathrm{K}$ complex, a resolving power exceeding 2000 is necessary 


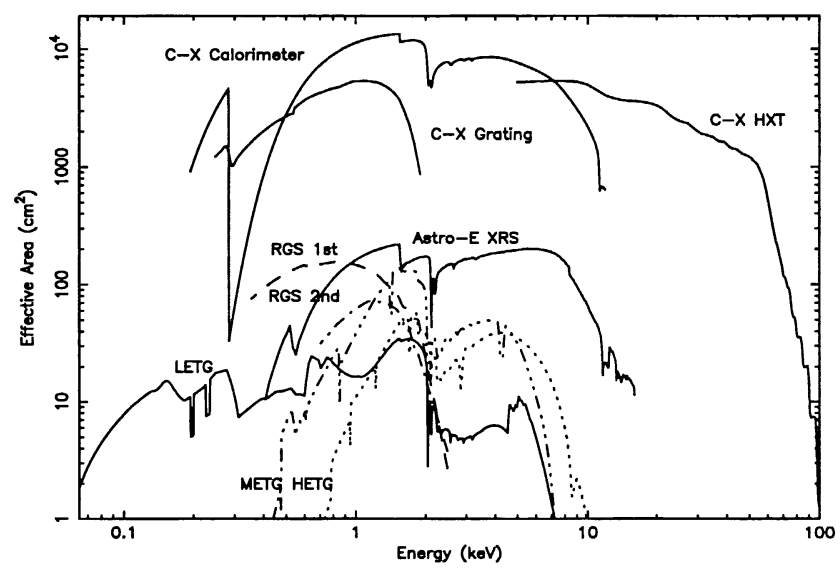

Figure 1. The effective area (shown as a log plot for clarity) of the three Constellation-X instruments (coaligned and operating simultaneously) for the four-satellite system. For comparison, the effective area of the high-resolution spectrometers on Chandra (METG, HETG, and LETG), XMM (RGS), and Astro-E (XRS) are shown.

to distinguish the lithium-like satellite lines from the overlapping helium-like transitions. Measurement of accurate radial velocities better than $100 \mathrm{~km} / \mathrm{s}$ from X-ray emission lines is central for many astrophysical investigations.

Spectra of high statistical quality in an observing time of $\leq 10^{5} \mathrm{~s}$ for large populations of sources at the faintest flux levels reached in the ROSAT deep surveys require an effective area 20-100 more than provided by the high-resolution spectrometers on Chandra, XMM, and Astro-E in the $0.25-10 \mathrm{keV}$ band. This corresponds to an effective area of $15,000 \mathrm{~cm}^{2}$ at $1 \mathrm{keV}$ and $6,000 \mathrm{~cm}^{2}$ at $6 \mathrm{keV}$ (including the spectrometer efficiency). The spectrometer efficiency is of order $50 \%$ at $1 \mathrm{keV}$, which corresponds to a raw telescope area of $30,000 \mathrm{~cm}^{2}$. Using a typical AGN spectrum an effective area of $1,500 \mathrm{~cm}^{2}$ at $40 \mathrm{keV}$ is required to match the $0.25-10 \mathrm{keV}$ band. A $>95 \%$ observing efficiency in a deep space orbit is assumed. For lower efficiency orbits, the effective area would have to be scaled up accordingly. The Constellation- $\mathrm{X}$ mission is designed to meet these requirements. The effective area curves of Constellation-X are shown in Figure 1 and compared with the equivalent curves from the high resolution spectrometers on Chandra, XMM, and Astro-E. The spectacular increase in capability over the previous missions is apparent.

\subsection{Mission Implementation}

The required large collecting area is achieved with a design utilizing several mirror modules, each with its own spectrometer/detector system. The Constellation$\mathrm{X}$ design (Figure 2) recognizes that several smaller spacecraft and more modest launch vehicles (e.g., Delta-class), each carrying one "science unit", can cost less than one very large spacecraft and launcher (e.g., Titan-class). The program is then very robust in that risks are distributed over several launches and spacecraft with no single failure leading to loss of mission. The baseline mission 
(GSFC/SAO)

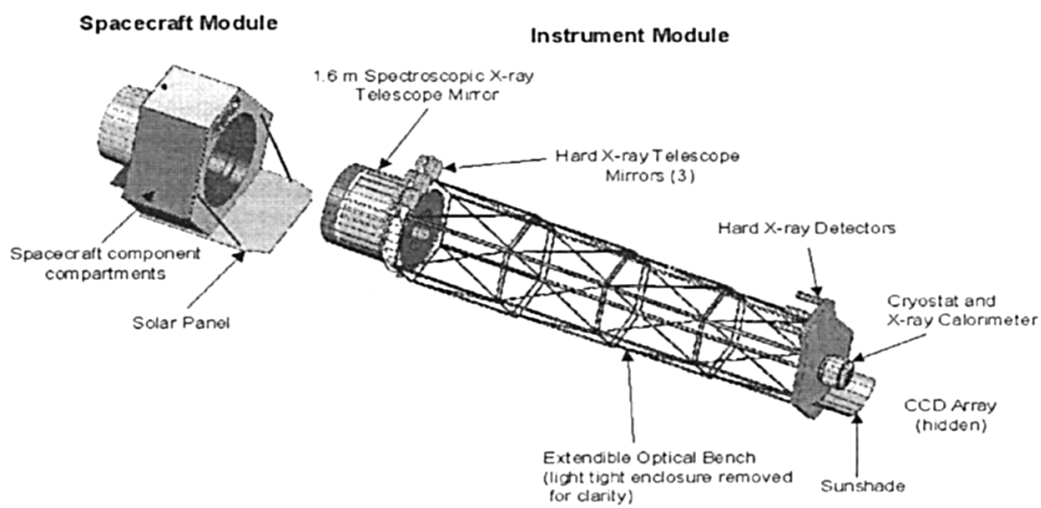

Figure 2. The current baseline GSFC-SAO design. There are four satellites in total, that are dual-manifested on two Atlas V or Delta IV launchers. Two satellites can be fit within the shroud by using an extendible optical bench to compress the (empty) length from the mirrors to the detectors. This is deployed on orbit to give the full focal length of $10 \mathrm{~m}$.

is four satellites that are carried in pairs on either two Atlas V or Delta IV launchers. The mission will be placed into a high earth or L2 orbit to facilitate high observing efficiency, provide an environment optimal for cryogenic cooling, and simplify the spacecraft design. The interval between the two launches will be of order 1 year. The mission lifetime with all four satellites on orbit will be $>4$ years. There will be minimal consumables, and, once on orbit, the mission lifetime can potentially last much longer.

\subsection{The Instruments}

To cover the entire $0.25-40 \mathrm{keV}$ band, Constellation-X will utilize a matched set of high-throughput focusing telescope systems. A spectroscopy X-ray telescope (SXT) covers the $0.25-10 \mathrm{keV}$ band and is optimized to maintain a spectral resolving power of at least 300 across the bandpass. The prime objective of the Constellation-X mission is X-ray spectroscopy, and, to do this, we must first and foremost maximize the number of photons collected. X-ray telescopes typically use a grazing incidence design and, because of this, resemble long, thin cylinders that are nested together. To maximize the collecting area per unit mass at minimal cost, we have selected an angular resolution requirement of at least 15 arcsec half-power diameter (HPD), with a goal of 5 arcsec. The $15 \operatorname{arcsec}$ HPD meets the confusion limit for the limiting flux of the sources to be studied.

The SXT uses two complementary spectrometer systems to achieve the desired energy resolution (Figure 1): an array of high-efficiency quantum microcalorimeters with energy resolution of $2 \mathrm{eV}$, and a set of reflection gratings. The gratings deflect part of the telescope beam away from the calorimeter array in a design similar to XMM, except that the Constellation-X direct beam falls on a high spectral-resolution quantum calorimeter instead of on a CCD. The field of 
view of the calorimeter is nominally 2.5 arcmin square, with 5 arcsecond pixels to adequately sample the telescope point spread function. The two spectrometers are complementary, with the grating optimal for high-resolution spectroscopy at low energies and the calorimeter optimal at higher energies (Figure 1). The gratings also provide coverage in the $0.3-0.5 \mathrm{keV}$ band where the calorimeter thermal and light-blocking filters cause a loss of response. This capability is particularly important for high-redshift objects, where line-rich regions will be moved down into this energy band. A $10^{5} \mathrm{~s}$ exposure of a typical AGN with the Constellation-X will obtain $\sim 1000$ counts in the calorimeter at a flux of $\sim$ $2 \times 10^{-15}$ c.g.s. $(0.2-2 \mathrm{keV}$ band), corresponding to the faintest sources detected in the deep ROSAT survey.

The Constellation-X calorimeter will provide a resolution of $2 \mathrm{eV}$ at $6 \mathrm{keV}$, which is a factor of 6 better than the device to be flown on Astro-E. This improvement in resolution, while not yet achieved, is well within the physical limitations of such devices. The recent advances in microcalorimetry have already achieved $\sim 2 \mathrm{eV}$ at $1 \mathrm{keV}$. The gratings and CCDs will be derived from the technology developed for Chandra and XMM. The challenge here is to light-weight the gratings and their assembly. In addition, the CCD detectors must be low power and also provide high quantum efficiency at low energies.

The Constellation-X hard X-ray telescope (HXT) uses multilayers to provide the first focusing optics system to operate in the $10-40 \mathrm{keV}$ band. The improvement in the signal to noise results in a factor of 100 or more increased sensitivity over nonfocusing methods used in this band. The HXT has a resolution of $\leq 1^{\prime}$ HPD. The GSFC-Nagoya Infocus and Caltech-Columbia HEFT balloon payloads will prove this approach in the next couple of years. The fine position resolution, along with the required efficiency and energy resolution ( $R$ $>10$ ), can be met by a cadmium zinc telluride (CZT) or Si strip detector system.

\section{Science Goals}

Constellation-X will be an observatory-class facility that will obtain detailed spectra from nearby stars out to AGN and clusters of galaxies at high redshift. The mission science falls into two main areas: 1) the study of matter under extreme gravity, in the vicinity of black holes; and 2) following the life cycles of matter in the evolving universe. In both areas, X-ray observations provide a key diagnostic tool. This is not surprising, since the virial temperature falls within the X-ray emitting band for a large range of diverse objects (stars, galaxies, clusters of galaxies). In essentially all cases where gravitational forces are important to the dynamics and energetics of the system, the presence of X-ray emitting gas is a natural consequence. As such, X-ray observations play a crucial role in addressing the origin, structure, and evolution of the Universe and of its principal material constituents: galaxies, stars, and dark matter.

\subsection{Supermassive Black Holes}

It is now generally accepted that quasars and related active galactic nuclei (AGN) are powered by accretion onto supermassive black holes, with masses of 1-1,000 million times that of the sun. These are the most energetic, nonexplosive energy sources in the Universe. Because of this, they were, for a long 
time, the most distant sources observable in the Universe and provide important clues to the formation and early evolution of galaxies. The relationship between the era of quasar activity, galaxy formation, and the evolution in the star formation rate is not understood. What has become clear over the past few years is that obscuration by dust and gas associated with star formation may be hiding much of the activity in the early universe. X-ray surveys provide a key capability to find AGN, especially those that are in dusty or obscured regions.

ROSAT has surveyed the sky and cataloged over 100,000 sources, and, in its deep field, resolved $\sim 80 \%$ of the X-ray background at $1 \mathrm{keV}$ (Hasinger et al. 1998). Optical identification programs reveal that many of the sources are AGN at a mean redshift of 1.5, and reaching out to redshift as high as 4 . Up to a million new AGN will be detected down to these flux levels by the XMM and Chandra serendipitous surveys. Over the past decade, we have seen that many of these AGN are heavily absorbed and that the central engine is visible only in hard X-rays. This observation is consistent with models for the Xray background above a few keV being dominated by absorbed AGN (Madau, Ghisellini, \& Fabian 1994) and has highlighted the importance of the hard (> $10 \mathrm{keV}$ ) X-ray band to study the total energy output of these objects. The total accretion energy released by AGN may be comparable to the energy generated by the stellar population, but with the AGN output mostly hidden behind obscuring starburst regions within 100 pc of the black hole (Fabian \& Iwasawa 1999).

The Constellation-X high-resolution spectrometers and hard X-ray sensitivity up to $40 \mathrm{keV}$ will be able to reach AGN populations at high redshift and determine the total energy output, as well as the geometry, ionization state, and density of the surrounding region. If most of accretion in the Universe is highly obscured, then the amount of emitted power per unit galaxy based on optical or UV quasar luminosity functions may have been under estimated (Fabian \& Iwasawa 1999). The hard X-ray band is relatively immune to this obscuration and allows a unique view of this early activity. Observations of AGN in the $\mathrm{X}$-ray band at $\mathrm{z}>1$ will hold important clues as to the overall evolution of the Universe (Figure 3).

The supermassive black holes in active galactic nuclei (AGN) represent the most extreme gravitational environment known, where the distortion of time and space predicted by General Relativity is most pronounced. A broad iron $\mathrm{K}$ line discovered by the ASCA Observatory by Tanaka et al. (1995) appears to be broadened by relativistic effects, indicating that it originates within a few gravitational radii of the black hole. This line provides an unexpected new diagnostic to determine not only the mass but also the spin of the black hole. Constellation-X is optimized to study this line feature in order to probe the region where strong gravity dominates, and to measure the spin and mass of the black hole for a large sample of objects over a wide range of redshift.

\subsection{Life Cycles of Matter in the Evolving Universe}

Models of galaxy formation aim to account for the properties of all structure, from the first dwarf galaxies to the clusters of galaxies, that are virializing now. Clusters of galaxies are the largest bound systems known, with their baryonic mass dominated by X-ray emitting plasma (which is ten times the mass of the sum of the constituent galaxies). X-ray observations are the best way to observe 


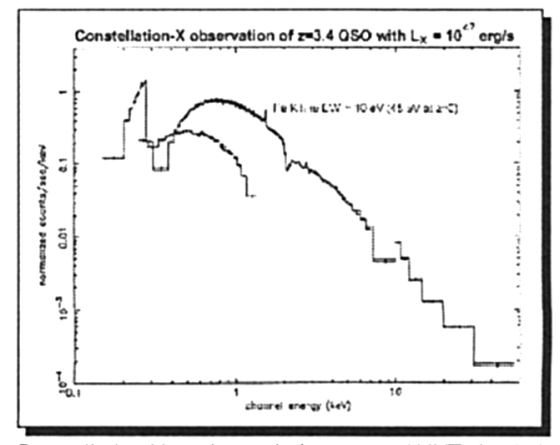

Constellation-X grating, calorimeter, and HXT simulation of a quasar at $z=3.2$.

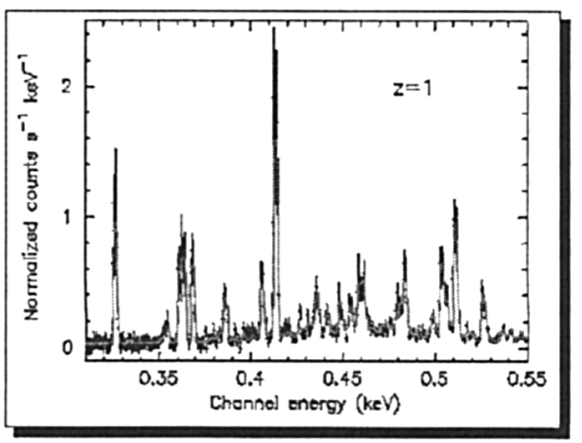

A 100 ks grating simulation of a starbursting Seyfert galaxy at $z=1$.

Figure 3. Two simulated Constellation-X observations of AGN at high redshift. On the left is a high-redshift quasar with (by today's standards) a very faint iron $\mathrm{K}$ line. This is easily detected by Constellation-X. On the right is a spectrum from a starburst galaxy at $\mathrm{z}=1$ that also hosts an AGN. X-ray observations are crucial to identify the relative contributions of starbursts vs. AGN activity. Constellation$\mathrm{X}$ is tuned to make these observations.

the formation and evolution of clusters (Mathiesen \& Evrard 1998). When and how these largest of structures came into existence are crucial tests of cosmological models.

Estimates of abundances derive from measurements of both the bremsstrahlung continuum and collisionally excited resonance emission lines. They trace the origins of stellar nucleosynthesis as we look back in redshift. Mapping the velocity distribution of this hot cluster gas via the Doppler shifts in the emission lines allows us to examine the dynamics within the cluster and, specifically, to study the effects of mergers between member galaxies and between separate clusters. More detailed constraints on the nature of the merger process are crucial to our understanding of the redistribution of the stellar processed material into the intracluster medium. X-ray spectroscopic observations also provide a direct means of mapping the dark matter content of clusters of galaxies and of understanding its relation to the baryonic tracers.

Constellation-X will be able to determine the internal velocity structure, gas fraction, and abundances of clusters and groups of galaxies out to high redshift (Figure 4). Constellation-X will test hierarchical clustering models by observing the first clusters as they start to virialize and by mapping the velocity and abundance distributions of nearby clusters and groups of galaxies. The observed baryons in the local universe fall far short of those predicted by standard Big Bang nucleosynthesis (Fukugita, Hogan, \& Peebles 1998). The baryons seen so clearly in the Lyman-alpha forest are not seen in the local universe. Numerical simulations by Cen \& Ostriker (1999) predict that most of these missing baryons 


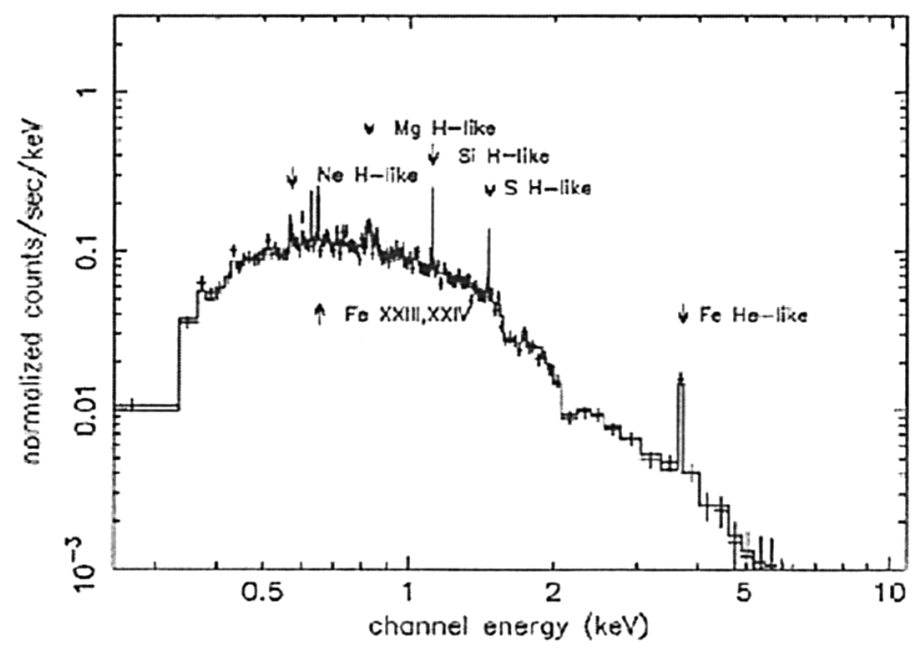

Figure 4. A simulated Constellation-X 50 ks observation of a cluster with a temperature of $4 \mathrm{keV}$ at a redshift of 0.8 . A type II supernova abundance distribution was assumed. The abundances of $\mathrm{Si}, \mathrm{S}$, and $\mathrm{Fe}$ are determined to $10 \%$ accuracy, and those of $\mathrm{Ne}$ and $\mathrm{Mg}$ to $20 \%$.

are in a hot intergalactic medium (IGM). The IGM should be detectable through $\mathrm{X}$-ray absorption lines imprinted by highly ionized metals on the spectrum of background quasars. Perna \& Loeb (1998) have shown that faint $(\mathrm{EW} \sim 0.1 \mathrm{eV})$ absorption lines can only be detected with the increased capability brought by Constellation-X.

One of the most important processes contributing to the chemical evolution of the Universe involves the release of stellar processed material into the interstellar medium via supernova explosions. Using Constellation-X, we will be able to detect line emission 100 times fainter than those seen by Chandra, XMM, or Astro-E. Consider the supernova W49B as an example. The ASCA CCD spectrum shows bright line complexes from He-like and $\mathrm{H}$-like ions of $\mathrm{Si}$, $\mathrm{S}, \mathrm{Ar}, \mathrm{Ca}$, and $\mathrm{Fe}$. In an observation of only $1,500 \mathrm{~s}$, Constellation-X will be able to detect (at the $10 \sigma$ level) and resolve the weakest of the He-like lines for these species. Furthermore, highly significant detections of the odd-Z elements in the group from $\mathrm{P}$ to $\mathrm{Ni}$ will be possible in $20 \mathrm{ks}$, while the weakest lines from the least abundant species ( $\mathrm{Sc}, \mathrm{V}, \mathrm{Co}, \mathrm{Cu}$, and $\mathrm{Zn}$ ) will be detected in $10^{5} \mathrm{~s}$.

The outermost layers of the atmospheres of normal stars form hot coronae confined by magnetic fields. X-ray spectroscopic observations determine the temperatures, densities, and geometries of these plasmas which then can be correlated with age, rotation, and other stellar parameters to better determine the mechanisms responsible for coronal heating. The detailed line spectra also provide abundance determinations which, in some cases, indicate differences from the abundances derived from optical spectra of the photosphere. Such abundance anomalies are not well understood, but they may be related to the processes by which photospheric material is "lifted" into the corona. With the resolution of Constellation-X, we will, for the first time, be able to 
directly measure the Doppler shift of X-ray lines to determine the location of the coronal structures within the binary systems. The spectroscopic capability of Constellation-X will also be able to study the coronae of nearby stars like our own sun, as well as the more luminous coronal systems in the LMC.

\section{Conclusion}

The Constellation-X technology development program is now well underway and is making tremendous progress. The current schedule allows for a new start in 2005 and launch in 2008/2009, about ten years after the launch of the Chandra X-ray Observatory. The goal is to have some overlap between these observatories to allow for both cross-calibration and a science synergy similar to that we currently see between HST and the Keck Observatory.

Acknowledgments. We thank Azita Valinia, Kim Weaver, Jean Grady, Oren Sheinman, Steve Kahn, Richard Mushotzky, Pat Tyler, and the entire Constellation-X Facility Science Team for their help with the preparation of this paper.

\section{References}

Cen, R., \& Ostriker, J. P. 1998, ApJ, 519, 109

Fabian, A. C., \& Iwasawa, K. 1999, MNRAS, 303, 34

Fukugita, M., Hogan, C. J., \& Peebles, P. J. E. 1998, ApJ, 500, 79

Hasinger, G., et al. 1998, A\&A, 329, 495

Madau, P., Ghisellini, G., \& Fabian, A. C. 1994, MNRAS, 270, L17

Mathiesen, B., \& Evrard, A. E. 1998, MNRAS, 295, 769

Perna, R., \& Loeb, A. 1998, ApJ, 503, 135

Reynolds, C. S., et al. 1999, ApJ, 514, 614

Tanaka, Y., et al. 1995, Nature, 375, 659 